\title{
New perspectives of immunomodulation and neuroprotection in glaucoma
}

\author{
PIOTR SKOPIŃSKII,2, DOROTA MAGDALENA RADOMSKA-LEŚNIEWSKA ${ }^{l}$, \\ JUSTYNA IZDEBSKA ${ }^{2,3}$, ANNA KAMINSKKA ${ }^{2,3}$, MAGDALENA KUPIS ${ }^{2,3}$, \\ ALEKSANDER JAN KUBIAK ${ }^{4}$, KATARZYNA SAMELSKA ${ }^{2,3}$ \\ ${ }^{1}$ Department of Histology and Embryology, Medical University of Warsaw, Warsaw, Poland \\ ${ }^{2}$ SPKSO Ophthalmic University Hospital, Warsaw, Poland \\ ${ }^{3}$ Department of Ophthalmology, Medical University of Warsaw, Warsaw, Poland \\ ${ }^{4}$ Rutgers New Jersey Medical School, Newark, NJ, United States
}

\begin{abstract}
Glaucoma is the neurodegenerative disease of retinal ganglion cells. The main risk factor for glaucoma is increased intraocular pressure. The processes leading to cell death due to presence of the injury factor comprise multiple molecular mechanisms, as well as the immunological response. The knowledge of immunological mechanisms occurring in glaucomatous degeneration makes it possible to introduce glaucoma treatment modulating the cellular degradation. The glaucoma treatment of the future will make it possible not only to lower the intraocular pressure, but also to moderate the intracellular mechanisms in order to prevent retinal cell degeneration. Citicoline is a drug modulating glutamate excitotoxicity that is already in use. Rho kinase inhibitors were found to stimulate neurite growth and axon regeneration apart from lowering intraocular pressure. The complementary action of brimonidine is to increase neurotrophic factor (NTF) concentrations and inhibit glutamate toxicity. Immunomodulatory therapies with antibodies and gene therapies show promising effects in the current studies. The supplementation of NTFs prevents glaucomatous damage. Resveratrol and other antioxidants inhibit reactive oxygen species formation. Cell transplantation of stem cells, Schwann cells and nerve extracts was reported to be successful so far. Our review presents the most promising new strategies of neuroprotection and immunomodulation in glaucoma.
\end{abstract}

Key words: brimonidine, growth factors, retinal ganglion cells (RGCs), Rho kinase inhibitors, stem cell therapy, citicoline.

(Cent Eur J Immunol 2021; 46 (1): 105-110)

\section{Introduction}

Glaucoma is a group of diseases characterized by progressive optic nerve degeneration. It leads to irreversible loss of retinal ganglion cells (RGCs) and their axons optic nerve fibers of the retina [1]. These changes can be observed by the thinning of the RGC layer and retinal nerve fiber layer of the retina.

Glaucoma is treated pharmacologically and surgically, decreasing the intraocular pressure. Surgical treatment is usually reserved until there is a failure of medical treatment. Topical therapy with ocular hypotensive drugs works by suppressing the production of the aqueous humor or by increasing the conventional or unconventional aqueous outflow pathway. The main anti-glaucoma topical medication classes are $\beta$-adrenergic antagonists, $\alpha 2$-adrenergic agonists, carbonic anhydrase inhibitors, prostaglandin $\mathrm{F}_{2 \mathrm{a}}$ analogues and, recently introduced, Rho kinase inhibitors [2, 3]. Out of these groups, the Rho kinase inhibitors are the only drugs resulting in decreased resistance in trabecular meshwork outflow by modifying the cytoskeleton of trabecular meshwork cells [4].

Increased intraocular pressure (IOP) has been known as a main factor of glaucoma incidence and progression. Targeting trabecular meshwork and aqueous humor outflow pathways has been the main strategy in glaucoma treatment. Ocular hypotensive drugs were proven effective in delaying or preventing glaucoma in patients with and without ocular hypertension [5-7].

Glaucoma is one of the leading causes of irreversible blindness in developed countries [8]. Its treatment was introduced during the second part of the $20^{\text {th }}$ century and has made great progress. Ocular hypotensive drugs are easily administered and widely available. In addition, there is a wide range of surgical procedures which effectively decrease intraocular pressure. However, achieving low

Correspondence: Katarzyna Samelska, Department of Ophthalmology, Medical University of Warsaw, 24/26 Marszałkowska St., 00-576 Warsaw, Poland, e-mail: samelskakatarzyna@gmail.com Submitted: 3.08.2020; Accepted: 21.09.2020 
intraocular pressure is often not enough to prevent glaucoma progression. Thus, ophthalmic research has become focused on understanding the molecular pathomechanisms in RGCs' glaucomatous degeneration.

The research in glaucoma pathophysiology has shown that numerous cellular mechanisms are involved in RGC loss. Since lowering IOP is often not enough to prevent glaucoma, there is a need for developing additional agents which allow retinal cells to withstand various pathological factors $[9,10]$.

The molecular basics of the glaucomatous damage include production of reactive oxygen species (ROS), deprivation of neurotrophic factors (NTFs), glial activation, excitotoxicity, ischemia and oxidative stress [11]. Those mechanisms are a possible target for neuroprotection and glaucoma treatment.

\section{Citicoline/glutamate excitotoxicity}

Glutamate is one of the main central nervous system (CNS) neurotransmitters. Glutamate acts on N-methylD-aspartate (NMDA) and non-NMDA receptors, activating proapoptotic cascades. Glutamate toxicity is considered a potential cause of glaucomatous damage. Increased glutamate concentration leads to ischemic insult in the CNS [12]. Memantine is an NMDA receptor antagonist blocking excessive glutamate activity [13] and is considered a protective agent in glaucoma therapy [12]. Clinical studies however suggest no effect on delaying visual field changes in patients receiving memantine treatment [14].

Citicoline is a nootropic drug and one of the neuroprotective agents used already in prevention of glaucomatous degeneration. Citicoline exerts its functions by counteracting glutamate excitotoxicity. Inhibition of glutamate action by citicoline reduces oxidative stress in RGC injury $[15,16]$.

Citicoline is a precursor for acetylcholine (ACh) the main neurotransmitter of the cholinergic system. The cholinergic signaling in CNS modulates visual processes. Moreover, the proper ACh metabolism is necessary for survival of the RGCs.

Citicoline crosses the blood-brain barrier after the breakdown to cytidine and choline. Upon entering the CNS, it is incorporated in biosynthesis of phosphatidylcholine, ACh, sphingomyelin and cardiolipin. It supports ACh formation and also serves as a rescue recourse for cellular membrane components [16]. These functions have been suggested to have the main role in citicoline neuroprotection in degenerative diseases of the CNS.

Citicoline is known for modulating the cholinergic, glutamatergic and dopaminergic neurotransmission in structures involved in glaucomatous degeneration. It has also been shown to maintain proper levels of sphingomyelin - one of the proteins involved in RGCs' axonal function and in effect enhance RGC survival [16].
Glaucoma treatment with citicoline causes delay in degenerative changes seen in electrophysiological testing (visual evoked potential). Citicoline is believed to halt glaucoma progression [15]. However, it cannot replace hypotensive treatment as its effect is transient and the drug would have to be given continuously to achieve a stable neuroprotective effect [15].

\section{Rho kinase inhibitors}

Rho kinase inhibitors are the newest class of clinically approved ocular hypotensive drugs. The class comprises ripasudil, which was approved in Japan in 2014, and netarsudil, which was approved in the United States in 2017. The Rho-associated coiled-coil-containing protein kinase (ROCK) family is the main effector of the Rho family and it comprises of ROCK 1 and ROCK2. These agents act on ROCK1 and ROCK2 Rho kinases in the trabecular meshwork [3]. Their main mechanism of action is attributed to lowering aqueous outflow resistance by modifying the myosin light chain in the cytoskeleton $[4,17]$. It also regulates neuroinflammation mediating the NF-kB pathways [3].

The effect of Rho kinase pathways on RGC degeneration has been investigated [18]. Apart from Rho kinase inhibitors' beneficial influence on IOP modulation, they present an additional neuroprotective effect on retinal neurons. ROCK inhibitors were shown to stimulate neurite growth and axonal regeneration [19]. Rho kinase pathways' suppression modulates and inhibits autophagy. Rho kinase inhibitors in experimental glaucoma models - administered topically as well as systemically - promoted RGC survival and regeneration [18, 20, 21]. The neuroprotective effect of Rho kinase inhibitors may play a supplementary role in glaucoma treatment.

\section{Brimonidine}

Brimonidine is an $\alpha 2$ adrenergic receptor agonist lowering intraocular pressure and thus preventing glaucoma progression. Inhibition of $\alpha 2$ adrenergic receptor results in increased aqueous outflow and decreased aqueous humor production [8]. Brimonidine was approved by the Food and Drug Administration for topical glaucoma treatment in 1996. It represents one of the main classes of topical ocular hypotensive agents.

Apart from its well-known action of lowering IOP, brimonidine has been reported to bear neuroprotective potential [13]. It has been reported to lead to elevation of NTFs $[22,23]$, reduce cyclic adenosine monophosphate (cAMP) concentrations and modulate NMDA receptors responsible for glutamate toxicity [8]. Amyloid $\beta$, known for the neurodegenerative pathway in Alzheimer's disease, has also been proposed to be involved in RGCs' glaucomatous injury. Brimonidine was reported to act on the amyloid- $\beta$ pathway and decrease its levels in an in vitro model [24], which explains brimonidine's additional neuroprotective function. 


\section{Statins}

Statins are agents used for systemic hypercholesterolemia. Their main action is to inhibit 3-hydroxy-3-methylglutaryl-coenzyme A reductase and suppress cholesterol synthesis. They additionally exert an anti-inflammatory effect through Rho kinase inhibition [25]. Apart from leading to cytoskeletal reorganization as well as cell shape changes in the trabecular meshwork and ciliary body in vitro [26], they show a protective effect on optic nerve head $(\mathrm{ONH})$ astrocytes [27]. Transforming growth factor $\beta 2$ (TGF- $\beta 2$ ) is a protein modulating cell differentiation, proliferation and chemotaxis. It also mediates extracellular matrix remodeling in $\mathrm{ONH}$ during glaucoma development. Statins' side-effect - TGF- $\beta 2$ inhibition - has a neuroprotective role in $\mathrm{ONH}$ changes in glaucomatous neurodegeneration [27].

\section{Immunomodulation}

Neuroinflammation plays a role in glaucomatous damage. The complement, tumor necrosis factor $\alpha$ (TNF- $\alpha$ ) and toll-like receptors (TLR) are shown to take part in pathways leading to RGC loss in animal and in vitro glaucoma models. The microglia and macroglia are involved in inflammatory responses to the injury signal. The proinflammatory cytokines exert an immunostimulatory effect and favor the interaction of glia with T lymphocytes, which are known for their neurodegenerative potential [28, 29].

Inhibition of TLR reduces astrocyte activation and the RGC death rate. The molecule TAK-242 (resatorvid) has been proven effective as a selective TLR4 inhibitor and neuroprotective agent in a murine glaucoma model [30].

It was found that glial response modulation with intravitreally administered ibudilast - a phosphodiesterase type 4 inhibitor - resulted in decreased secretion of proinflammatory mediators and activation of the cAMP/PKA pathway, which in effect enhanced RGC survival [31].

The complement pathway includes activity of proteins $\mathrm{C} 1, \mathrm{C} 3$ and $\mathrm{C} 5$, which promotes membrane attacking complex formation and cell lysis. The increased activity of complement has been found in eyes in animal glaucoma models [32-35]. Complement inhibitor therapies are in preclinical and clinical trial phases for age-related macular degeneration [36]. As for glaucoma, the murine model trial of CR2-Crry gene therapy affecting complement showed promising results [37]. The gene CR2-Crry codes Crry - the main regulator of $\mathrm{C} 2$ involved in the complement pathway. Treated retinas showed overexpression of Crry, which resulted in inhibition of the complement pathway, leading to reduction of the RGC degeneration rate [37]. Intravitreal therapy with antibodies suppressing complement pathways also showed beneficial results. The trial with the C5-I-C5 complement component antibody prevented the loss of retinal cells [38].
Fas ligand (FasL) promotes the extrinsic apoptotic pathway by binding to the Fas/CD95 trans-membrane receptor. As a result, the caspase cascade is activated [39]. Studies investigating inhibition of FasL-Fas by a small peptide of the Fas receptor antagonist named ONL1204 showed neuroprotective and immunomodulatory effects [40]. Fas receptor inhibition reduced gliosis and macrophage infiltration and decreased the concentration of proinflammatory cytokines and chemokines, such as TNF- $\alpha$, interleukin (IL)-1 $\beta$, glial fibrillary acidic protein (GFAP), caspase 8, TLR4 and C3 and C1Q complement components. The treatment of glaucomatous eyes with ONL1204 prevented axon degeneration and resulted in decrease of the RGC death rate.

TNF- $\alpha$ is a proinflammatory cytokine playing a role in glaucomatous degeneration. It promotes mitochondrial cell death pathways and induces ROS generation. The systemic administration of the Food and Drug Administration (FDA)-approved anti-TNF- $\alpha$ antibody etanercept showed the response in glaucomatous retinas. In glaucoma models, eyes treated with etanercept showed reduced microglial activation and degeneration of RGCs' axons and somas [41].

\section{Neurotrophic factors}

Neurotrophic factors exert various effects by binding to different receptors. They act on development and survival of neurons. They mainly promote cell survival by activating tropomyosin receptor kinase (Trk) surface receptors, as well as inducing apoptosis on interaction with the p75 TR receptor [11].

Brain derived neurotrophic factor (BDNF) is produced in the superior colliculus and lateral geniculate nucleus as well as locally by RGCs and retinal astrocytes. It promotes RGC survival through stimulating the extracellular signal-regulated kinases (Erk) Erk1/2 and c-jun and suppressing caspase 2. Brain derived neurotrophic factor is produced throughout the body, locally by RGCs and also in the brain. It is transported to the retina in the retrograde axonal transport [42, 43]. The $\alpha 2$-adrenergic agonist brimonidine was found to increase BDNF expression in RGCs and thus cause a neuroprotective effect [23]. The NTFs are known for stimulating prosurvival, as well as the proapoptotic pathways - such as c-Jun N-terminal cascade. Their mechanisms of action affect diverse receptors and multiple pathways.

It has been found that ciliary neurotrophic factor along with glial cell-derived neurotrophic factor intravitreal administration may synergistically promote RGC survival in optic nerve crush models [44]. Exogenous administration of the NTFs has been proven to promote RGC survival in glaucoma models but works only temporarily, supposedly due to downregulation of TrkB receptors [45, 46].

Apart from NTF administration, other projects have focused on stimulating NTF production. Stimulation of the superior colliculus utilizing an optogenetic approach led to 
an increase in BDNF production and reduced RGC loss in a murine glaucoma model $[45,47]$.

In vitro and in vivo murine models utilizing gene therapy to stimulate the BDNF/TrkB pathway have potential in producing long-term neuroprotective effects. The models use the adeno-associated virus to introduce the sequence coding both BDNF and TrkB receptors in a single transgene. The therapy results in stimulated BDNF production but also provides continuous signaling by causing increased expression of TrkB in the inner retina [46].

The neurotrophic factor deprivation theory is considered to be a crucial part of cellular pathology mechanisms of RGC death [11, 43]. However, therapies targeting NTF supplementation have not been fully successful. The exact action of particular NTFs and NTF retrograde axonal transportation is still unknown. This knowledge would enable us to fully comprehend the pathways involved in NTF deprivation in the retinal cells [48].

\section{Antioxidants}

Reactive oxygen species have a neurotoxic effect on the retinal cells. Reactive oxygen species result in glial cell activation which causes damage to the ONH. The experimental glaucoma treatment consisting of subcutaneously administered Tempol (4-hydroxytetramethylpiperidine-1-oxyl) - a multifunctional antioxidant - resulted in decreased NF-kB activity in RGCs. In effect, the antioxidative agent suppresses the inflammatory response and neurodegeneration in the glaucoma ischemic model [49]. In conclusion, Tempol therapy shows promising results in glaucoma treatment and prevention.

Resveratrol (RSV) acts as an activator of sirtuin 1, which regulates cell senescence and apoptosis. Resveratrol has been shown to have antioxidative properties and have beneficial effects in human organ injuries [50]. It has been shown that intravitreal RSV injections inhibited ROS formation, resulting in a neuroprotective effect on RGCs, downregulated acetyl-p53 protein formation and caused an increase in BDNF expression in the high-IOP murine glaucoma model [50]. Another study showed that intraperitoneal RSV treatment in the experimental rat glaucoma model caused RGC loss delay via inhibition of proapoptotic action of Bax proteins. It also suppressed reactive gliosis and led to an increase in GFAP level [51]. The antioxidative and anti-inflammatory effects of RSV bear potential for the future neuroprotection of retinal cells.

\section{Cell transplantation treatment}

\section{Stem cell therapy}

Intravitreal cell transplantation is an important field of glaucoma neuroprotection research. Cell replacement provides the nutrients to areas of injury by replacing the degenerated cells with new ones. The stem cell therapy includes transplantation of human pluripotent stem-cell derived RGCs and mesenchymal stem cells (MSC) [8]. Mesenchymal stem cell therapy has the potential to be a future glaucoma treatment [52]. Mesenchymal stem cells are reported to have the ability to create functional RGC-like cells in the site of injury $[53,54]$. They also have therapeutic potential through the capacity to secrete exosomes (Exos) which may act as carriers for proteins, e.g. neurotrophins [42]. The intravitreally injected Exos reside in proximity to the retina for more than four weeks and provide the continuous delivery of neurotrophins, which resulted in the reduction in RGCs and axon death $[55,56]$.

Stem cells also produce numerous factors promoting cell survival, e.g. NTFs such as platelet-derived growth factor (PDGF), BDNF, nerve growth factor (NGF) and ciliary neurotrophic factor (CNTF) $[8,42]$. Some authors suggest that PDGF is the key mesenchymal stem/stromal cell (MSC)-derived molecule crucial for the RGC survival [57]. Mesenchymal stem/stromal cell administration was found to be neuroprotective by stimulating production of high levels of growth factors. However, it has also been found to cause some adverse effects such as reactive gliosis, vitreous clamping and epiretinal membrane formation [58]. Chen et al. stated that the umbilical cord blood MSCs' neuroprotective effect on RGCs is transient and does not prevent cell death after cessation of the therapy [59].

Attempts of experimental animal therapy with genetically modified MSC overexpressing BDNF and NGF were successful. Modified MSC therapy resulted in increased RGC survival and regeneration compared to therapy with unmodified MSC $[53,60]$. Further action to prevent downregulation of TrkB receptors in NTF therapy has to be considered $[45,46]$.

\section{Schwann cells}

Schwann cells (SC) have a beneficial effect on RGCs in an in vivo model. The SC homogenate without NTFs such as NGF, BDNF, CNTF, NT3 has been administered in retinal insert explants and intravitreal injections. It was found to have a positive effect for RGC protection and regeneration as axon regrowth, in spite of the absence of NTF supplementation [61].

\section{Nerve extracts}

The pre-degenerated sciatic nerve extract administered as intravitreal injections in a mouse experimental glaucoma model was revealed to stimulate the outgrowth of damaged neurites in the CNS and protect RGCs from death [62]. Among the contents of this extract, MT2 (metallothionein 2) has been found to be the key neuroprotective molecule. MT2 induces endogenous BDNF expression and reduces expression of proapoptotic molecules such as $\mathrm{p} 38$, IL-6, IL-1b and TNF- $\alpha$ [63]. 


\section{Conclusions}

As of today, lowering the IOP is the main glaucoma therapy target. Neuroprotection remains a developing strategy which is already finding a place in modern treatment. The neuroprotective agent citicoline is currently used both topically and systemically. Promotion of RGC survival as a side-effect of Rho-kinase inhibitors, brimonidine and statins seems to be beneficial. The research targeting various steps of apoptosis pathways and targeting alterations in molecular mechanisms of retinal neurons shows high potential for lowering the glaucoma progression rate. The discovery of the immune system's role in glaucoma pathogenesis allows for targeting immunomodulation in preventing glaucomatous damage.

Glaucoma is a group of heterogenous diseases and no specific genetical cause for retinal cell loss has been found. However, gene therapies affecting production of molecules taking part in the pathways of neurodegeneration are promising. Neurotrophic factor supplementation provides easy intravitreal administration of the medication and has had satisfactory results in mice. Nonetheless, the regulation of NTF receptors has to be studied to determine the long-lasting effect of the treatment. Antioxidative agents have also been shown effective in modulating cell death pathways. Another powerful approach to glaucoma therapy is cell replacement therapy. Like in other neurodegenerative conditions, pluripotential cells and stem cells have the potential to replace the neurons lost due to glaucoma.

\section{The authors declare no conflict of interest.}

\section{References}

1. Weinreb RN, Aung T, Medeiros FA (2014): The pathophysiology and treatment of glaucoma: a review. JAMA 311: 19011911.

2. Conlon R, Saheb H, Ahmed II (2017): Glaucoma treatment trends: a review. Can J Ophthalmol 52: 114-124.

3. Tanna AP, Johnson M (2018): Rho Kinase Inhibitors as a novel treatment for glaucoma and ocular hypertension. Ophthalmology 125: 1741-1756.

4. Pattabiraman PP (2015): Effects of Rho kinase inhibitor AR-13324 on the actin cytoskeleton and on TGF $\beta 2$ - and CTGF-induced fibrogenic activity in Human Trabecular Meshwork Cells. AOPT 2015 Poster 43.

5. Kass MA, Heuer DK, Higginbotham EJ, et al. (2002): The ocular hypertension treatment study: a randomized trial determines that topical ocular hypotensive medication delays or prevents the onset of primary open-angle glaucoma. Arch Ophthalmol 120: 701-713.

6. Heijl A, Leske MC, Bengtsson B, et al. (2002): Reduction of intraocular pressure and glaucoma progression: results from the Early Manifest Glaucoma Trial. Arch Ophthalmol 120: 1268-1279.

7. Garway-Heath DF, Lascaratos G, Bunce C, et al. (2013): The United Kingdom Glaucoma Treatment Study: a multicenter, randomized, placebo-controlled clinical trial: design and methodology. Ophthalmology 120: 68-76.
8. Fu L, Kwok SS, Chan YK, et al. (2019): Therapeutic strategies for attenuation of retinal ganglion cell injury in optic neuropathies: concepts in translational research and therapeutic implications. Biomed Res Int 2019: 8397521.

9. Tasneem Z, Martin K, Martin KR (2020): Neuroprotection in glaucoma: towards clinical trials and precision medicine. Curr Eye Res 45: 327-338.

10. Almasieh M, Levin AL (2017): Neuroprotection in glaucoma: animal models and clinical trials. Annu Rev Vis Sci 3: 91-120.

11. Pietrucha-Dutczak M, Amadio M, Govoni S, et al. (2018): The role of endogenous neuroprotective mechanisms in the prevention of retinal ganglion cells degeneration. Front Neurosci 12: 834 .

12. Hare WA, Wolde Mussie E, Lai RK, et al. (2004): Efficacy and safety of memantine treatment for reduction of changes associated with experimental glaucoma in monkey, I: functional measures. Invest Ophthalmol 45: 2625-2639.

13. Boia R, Ruzafa N, Aires ID, et al. (2020): Neuroprotective strategies for retinal ganglion cell degeneration: current status and challenges ahead. Int J Mol Sci 21: 2262.

14. Weinreb RN, Liebmann JM, Cio GA, et al. (2018): Oral memantine for the treatment of glaucoma. Ophthalmology 125: 1874-1885.

15. Grieb P, Jünemann A, Rekas M, et al. (2016): Citicoline: a food beneficial for patients suffering from or threated with glaucoma. Front Aging Neurosci 8: 73.

16. Faiq MA, Wollstein G, Schuman JS, et al. (2019): Cholinergic nervous system and glaucoma: From basic science to clinical applications. Prog Retin Eye Res 72: 100767.

17. Inoue T, Tanihara H, Tokushige H, et al. (2015): Efficacy and safety of SNJ-1656 in primary open-angle glaucoma or ocular hypertension. Acta Ophthalmol 2015: e393-5.

18. Koch JC, Tonges L, Barski E, et al. (2014): ROCK2 is a major regulator of axonal degeneration, neuronal death and axonal regeneration in the CNS. Cell Death Dis 5: e1225.

19. Dergham P, Ellezam B, Essagian C, et al. (2002): Rho signaling pathway targeted to promote spinal cord repair. J Neurosci 22: 6570-6577.

20. Shaw PX, Sang A, Wang Y, et al. (2017): Topical administration of a Rock/Net inhibitor promotes retinal ganglion cell survival and axon regeneration after optic nerve injury. Exp Eye Res 158: 33-42.

21. Yamamoto K, Maruyama K, Himori N, et al. (2014): The novel Rho kinase (ROCK) inhibitor K-115: a new candidate drug for neuroprotective treatment in glaucoma. Invest Ophthalmol Vis Sci 55: 7126-7136.

22. Degos V, Charpentier TL, Chhor V, et al. (2013): Neuroprotective effects of dexmedetomidine against glutamate agonist-induced neuronal cell death are related to increased astrocyte brain-derived neurotrophic factor expression. Anesthesiology 118: 1123-1132.

23. Gao H, Qiao X, Cantor LB, et al. (2002): Upregulation of brain-derived neurotrophic factor expression by brimonidine in rat retinal ganglion cells. Arch Ophthalmol 120: 797-803.

24. Nizari S, Guo L, Davis BM, et al. (2016): Non-amyloidogenic effects of $\alpha 2$ adrenergic agonists: implications for brimonidine-mediated neuroprotection. Cell Death Dis 7: e2514.

25. Nohria A, Prsic A, Liu PY, et al. (2009): Statins inhibit Rho kinase activity in patients with atherosclerosis. Atherosclerosis 205: 517-521.

26. Song J, Deng PF, Stinnett SS, et al. (2005): Effects of cholesterol-lowering statins on the aqueous humor outflow pathway. Invest Ophthalmol Vis Sci 46: 2424-2432.

27. Kim ML Sung KR, Shin JA, et al. (2017): Statins reduce TGF-beta2-modulation of the extracellular matrix in cultured astrocytes of the human optic nerve head. Exp Eye Res 164: 55-63. 
28. Bariş M, Tezel G (2019): Immunomodulation as a neuroprotective strategy for glaucoma treatment. Curr Ophthalmol Rep 7: 160-169.

29. Bell K, Hohenstein-Blaul NVT, Teister J, et al. (2018): Modulation of the immune system for the treatment of glaucoma. Curr Neuropharmacol 16: 942-958.

30. Nakano Y, Shimazawa M, Ojino K, et al. (2017): Toll-like receptor 4 inhibitor protects against retinal ganglion cell damage induced by optic nerve crush in mice. J Pharmacol 133: 176-183.

31. Cueva Vargas JL, Belforte N, Di Polo A (2016): The glial cell modulator ibudilast attenuates neuroinflammation and enhances retinal ganglion cell viability in glaucoma through protein kinase A signaling. Neurobiol Dis 93: 156-171.

32. Kuehn MH, Kim CY, Ostojic J, et al. (2006): Retinal synthesis and deposition of complement components induced by ocular hypertension. Exp Eye Res 83: 620-628.

33. Becker S, Reinehr S, Burkhard Dick H, et al. (2015): Complement activation after induction of ocular hypertension in animal model. Ophthalmologe 112: 41-48.

34. Soto I, Howell GR (2014): The complex role of neuroinflammation in glaucoma. Cold Spring Harb Perspect Med 4: a017269.

35. Williams PA, Tribble JR, Pepper KW, et al. (2016): Inhibition of the classical pathway of the complement cascade prevents early dendritic and synaptic degeneration in glaucoma. Mol Neurodegener 11: 26.

36. Xu H, Chen M (2016): Targeting the complement system for the management of retinal inflammatory and degenerative diseases. Eur J Pharmacol 787: 94-104.

37. Bosco A, Anderson SR, Breen KT, et al. (2018): Complement C3-targeted gene therapy restricts onset and progression of neurodegeneration in chronic mouse glaucoma. Mol Ther 26: 2379-2396.

38. Reinehr S, Gomes SC, Gassel CJ, et al. (2019): Intravitreal therapy against the complement factor $\mathrm{c} 5$ prevents retinal degeneration in an experimental autoimmune glaucoma model. Front Pharmacol 10: 1381.

39. Almasieh M, Wilson AM, Morquette B, et al. (2012): The molecular basis of retinal ganglion cell death in glaucoma. Prog Retin Eye Res 31: 152-181.

40. Krishnan A, Kocab AJ, Zacks DN, et al. (2019): A small peptide antagonist of the Fas receptor inhibits neuroinflammation and prevents axon degeneration and retinal ganglion cell death in an inducible mouse model of glaucoma. J Neuroinflammation 16: 184

41. Roh M, Zhang Y, Murakami Y, et al. (2012): Etanercept, a widely used inhibitor of tumor necrosis factor-alpha (TNF-alpha), prevents retinal ganglion cell loss in a rat model of glaucoma. PLoS One 7: e40065.

42. Harrell CR, Fellabaum C, Arsenijevic A, et al. (2019): Therapeutic potential of mesenchymal stem cells and their secretome in the treatment of glaucoma. Stem Cells Int 27: 7869130.

43. Johnson TV, Bull ND, Martin KR (2011): Neurotrophic factor delivery as a protective treatment for glaucoma. Exp Eye Res 93: 196-203.

44. Flachsbarth K, Jankowiak W, Kruszewski K, et al. (2018): Pronounced synergistic neuroprotective effect of GDNF and CNTF on axotomized retinal ganglion cells in the adult mouse. Exp Eye Res 176: 258-265.

45. Dekeyster E, Geeraerts E, Buyens T, et al. (2015): Tackling glaucoma from within the brain: an unfortunate interplay of BDNF and TrkB. PLoS One 11: e0142027.

46. Osborne A, Wang AXZ, Tassoni A, et al. (2018): Design of a novel gene therapy construct to achieve sustained brain-de- rived neurotrophic factor signaling in neurons. Hum Gene Ther 29: 828-841.

47. Geeraerts E, Claes M, Dekeyster E, et al. (2019): Optogenetic stimulation of the superior colliculus confers retinal neuroprotection in a mouse glaucoma model. J Neurosci 39: 2313-2325.

48. Claes M, De Groef L, Moons L (2019): Target-derived neurotrophic factor deprivation puts retinal ganglion cells on death row: cold hard evidence and caveats. Int J Mol Sci 20: 4314.

49. Yang X, Hondur G, Tezel G (2016): Antioxidant treatment limits neuroinflammation in experimental glaucoma. Invest Ophthalmol Vis Sci 57: 2344-2354.

50. Cao K, Ishida T, Fang Y, et al. (2020): Protection of the retinal ganglion cells: intravitreal injection of resveratrol in mouse model of ocular hypertension. Invest Ophthalmol Vis Sci 61: 13.

51. Luo H, Zhuang J, Hu P, et al. (2018): Resveratrol delays retinal ganglion cell loss and attenuates gliosis-related inflammation from ischemia-reperfusion injury. Invest Ophthalmol Vis Sci 59: 3879-3888.

52. Emre E, Yüksel N, Duruksu G, et al. (2015): Neuroprotective effects of intravitreally transplanted adipose tissue and bone marrow - derived mesenchymal stem cells in an experimental ocular hypertension model. Cytotherapy 17: 543-559.

53. Harrell CR, Simovic Markovic B, Fellabaum C, et al. (2018): Therapeutic potential of mesenchymal stem cell-derived exosomes in the treatment of eye diseases. Adv Exp Med Biol 1089: 47-57.

54. Roozafzoon R, Lashay A, Vasei M, et al. (2015): Dental pulp stem cells differentiation into retinal ganglion-like cells in a three dimensional network. Biochem Biophys Res Commun 6: 154-160.

55. Mead B, Tomarev S (2017): Bone marrow-derived mesenchymal stem cells-derived exosomes promote survival of retinal ganglion cells through miRNA-dependent mechanisms. Stem Cells Transl Med 6: 1273-1285.

56. Mead B, Ahmed Z, Tomarev S (2018): Mesenchymal stem cell-derived small extracellular vesicles promote neuroprotection in a genetic DBA/2J mouse model of glaucoma. Invest Ophthalmol Vis Sci 59: 5473-5480.

57. Johnson TV, De Korver NW, Levasseur VA, et al. (2014): Identification of retinal ganglion cell neuroprotection conferred by platelet-derived growth factor through analysis of the mesenchymal stem cell secretome. Brain 137: 503-519.

58. Khatib TZ, Martin KR (2020): Neuroprotection in glaucoma: towards clinical trials and precision medicine. Curr Eye Res 45: 327-338.

59. Chen M, Xiang Z, Cai J (2013): The anti-apoptotic and neuro-protective effects of human umbilical cord blood mesenchymal stem cells (hUCB-MSCs) on acute optic nerve injury is transient. Brain Res 1532: 63-75.

60. Harper MM, Grozdanic SD, Blits B, et al. (2011): Transplantation of BDNF-secreting mesenchymal stem cells provides neuroprotection in chronically hypertensive rat eyes. Invest Ophthalmol Vis Sci 52: 4506-4515.

61. Smedowski A, Liu X, Pietrucha-Dutcak M, et al. (2016): Predegenerated Schwann cells - a novel prospect for cell therapy for glaucoma: neuroprotection, neuroregeneration and neuroplasticity. Sci Rep 6: 23187.

62. Gołka B, Lewin-Kowalik J, Swiech-Sabuda E, et al. (2001): Predegenerated peripheral nerve grafts rescue retinal ganglion cells from axotomy-induced death. Exp Neurol 167: 118-125.

63. Pietrucha-Dutczak M, Smedowski A, Liu X, et al. (2017): Candidate proteins from predegenerated nerve exert time-specific protection of retinal ganglion cells in glaucoma. Sci Rep 7: 14540. 